\title{
Hollow Core Optical Fibers for Industrial Ultra Short Pulse Laser Beam Delivery Applications
}

\author{
Sebastian Eilzer* and Björn Wedel \\ PT Photonic Tools GmbH, Johann-Hittorf-Straße 8, 12489 Berlin, Germany; b.wedel@photonic-tools.de \\ * Correspondence: s.eilzer@photonic-tools.de; Tel.: +49-30-6392-78000
}

Received: 14 September 2018; Accepted: 10 October 2018; Published: 16 October 2018

\begin{abstract}
Hollow core fibers were introduced many years ago but are now starting to be used regularly in more demanding applications. While first experiments mainly focused on the characterization and analysis of the fibers themselves, they are now implemented as a tool in the laser beam delivery. Owing to their different designs and implementations, different tasks can be achieved, such as flexible beam delivery, wide spectral broadening up to supercontinuum generation or intense gas-laser interaction over long distances. To achieve a constant result in these applications under varying conditions, many parameters of these fibers have to be controlled precisely during fabrication and implementation. A wide variety of hollow core fiber designs have been analyzed and implemented into a high-power industrial beam delivery and their performance has been measured.
\end{abstract}

Keywords: hollow core fiber; beam delivery; ultrafast lasers

\section{Introduction}

Laser sources with ultrashort pulses have taken a rapid development and broader industrial use over the past couple of years. Of particular interest are material processing technologies, which enable manufacturing of highly precise structures and delicate materials [1]. Thanks to the short light-matter interaction of ultrafast laser pulses, thermal damage of material substrates can be greatly reduced and also new interactions, such as filamentation, can be used.

While the development of laser sources and material processing technologies have steadily evolved (both in terms of available optical parameters as well as industrial performance), system technology has often been identified to be the limiting factor in today's industrial application. This includes beam transport and beam steering and shaping.

The usage of continuous-wave high-power lasers in the industry has made a huge leap forward by incorporating flexible fiber-based beam delivery. There are many advantages of using a fiber beam transport, compared to free space, especially in an industrial environment. The most obvious advantage is the flexible use of the laser at different positions while maintaining the beam properties. While gantry units can be realized with stabilized free-space setups, the option for different angles and flexibility with a fiber are far greater, especially in robot arm applications. These advantages are made accessible for the use of high-power ultrafast lasers by the introduction of hollow core fiber. Their laser beams cannot be transported with traditional step-index fibers as their peak intensity lies above the damage threshold of silica and would therefore destroy the fiber itself. Thanks to the air- or gas-filled core in a hollow core fiber, it is possible to efficiently guide short laser pulses with little dispersion and high peak power over relevant distances.

The first microstructured hollow core fibers were reported by Cregan et al. in 1999 [2]. To contain the laser energy inside a hollow core, distinctly different physical phenomena, compared to those of step-index fibers, have to be applied, since the material surrounding the core has a higher refractive index. The ordered and repetitive structure of a bandgap fiber cladding allows for wavelength areas 
where the light does not couple into the cladding and therefore remains inside the core. This basic concept, explained in different ways and with varying models, still holds true for any hollow core fiber design.

The integration of hollow core optical fibers into laser light cables has been presented by Photonic Tools in 2015. The ultrafast beam delivery comprises a coupling unit, the laser light cable itself and a modular processing head (see Section 2.3) and can be used for applications ranging from sensing to material processing or nonlinear pulse shaping.

The aim of this work is to summarize the integration of hollow core fiber in an industrial environment and present some representative applications.

\section{Materials and Methods}

\subsection{Types of Fibers}

There exists a large variety of hollow core fibers which could in principle be used for transmission of ultrafast laser pulses. However, when considering factors such as required lengths, typical laser application parameters, mechanical tolerances, optical layouts and operation environments, quite a few new boundary conditions play a role in designing the optimal fiber.

A key point, when working with hollow core fibers, concerns the existence of more than one mode inside the core. The amount of dampening of each of these modes determines beam quality at the output when several modes are excited, either during coupling into the fiber or when disturbances influence the transmission. If higher-order modes are highly suppressed, a lower overall transmission can be expected. To transmit higher pulse energies and couple them reliably into a fiber, a larger core is necessary; however, a larger core also allows for less separation between the higher-order modes inside the fiber, leading to possible instability.

To still maintain a high beam quality, special care has to be taken when designing the coupling optics to excite mostly the fundamental mode of the fiber. To contain the light inside the core and reduce coupling into the cladding, core geometries with a negative curvature have been found to be very effective in keeping the high field strength away from the surrounding glass structure [3]. These fibers offer a low loss over a wide wavelength range while still maintaining larger core sizes. There are two main types which are called Kagome fibers, due to the lattice structure, and antiresonant fibers. More well-known are single-ring or revolver fibers which build up the core region by surrounding it with one layer of non-touching rings, or cone-shaped structures (see Figure 1).
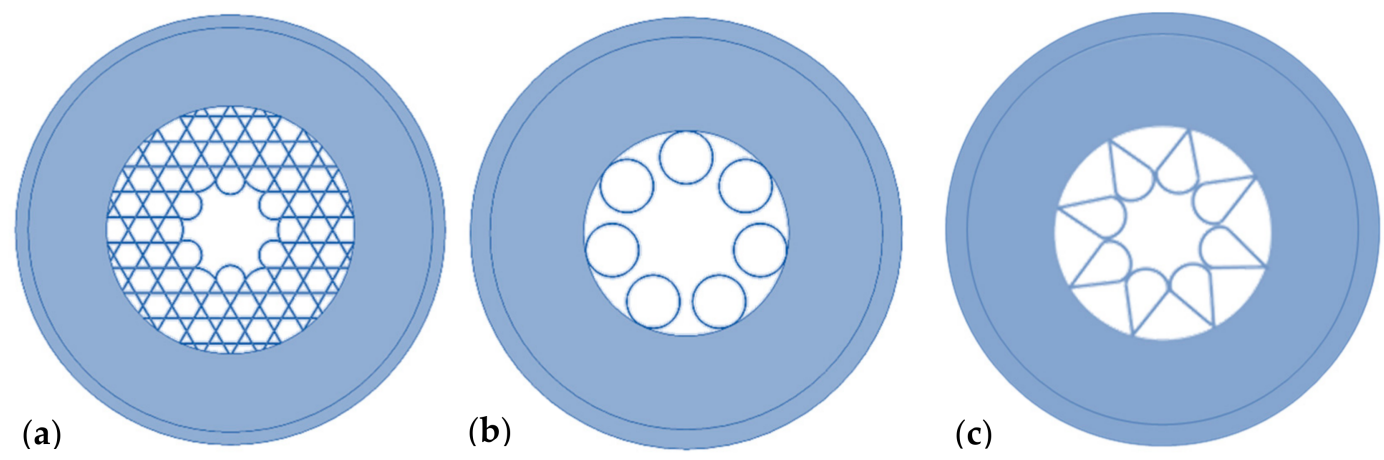

Figure 1. Depictions of common large-area hollow core fiber designs: (a) Kagome-type fiber; (b) single ring- or revolver-type fiber; and (c) cone-type fiber.

In order to determine the spectral properties and dynamic behavior of these fibers, complex studies of their guiding mechanism are required. Many models of varying complexity have been proposed and compared to analytical calculations as well as experiments. Influences on confinement by the wall thickness [4,5] and by the cladding structure [6] have been investigated, as well as dispersion of these fibers [7]. When taking industrial use into consideration, additional influences on the beam properties, 
e.g., by bending the fiber, have to be included to achieve an optimized design [8,9]. Therefore, by tuning fiber structure paramters, like core size, web thickness and structure elements, the fibers can be tailored for specific purposes and applications such as spectral broadening up to supercontinuum generation [10] or intense gas-laser interaction over long distances [11].

\subsection{Limitations of the Technology}

With increasing beam power, one has to look at the limits a hollow core beam transport possesses, determined by the physics involved. These include the maximum energy of lasers pulses and the shortest possible pulse length.

The maximum energy transmission in a hollow core fiber is still limited by the destruction of the silica [12]. The silica structure is not in the center of the beam where the energy density is highest, but rather in the outer areas. It is important to note that while the fundamental mode of the fiber has only very little overlap with the structure, the critical point is coupling into the fiber. The fundamental free-space mode has to excite the fundamental fiber mode, and the matched free-space Gaussian mode has a slightly higher overlap with the unmodified fiber structure than the fundamental fiber mode. A small portion of light will overlap directly with the glass struts of the fiber and interact with it. Using this assumption, one can determine a peak power dependent on the core size and the matched beam that is coupled into it (see Figure 2). In real-life applications, a reduced coupling accuracy and slight movement of the beam have to be taken into account.

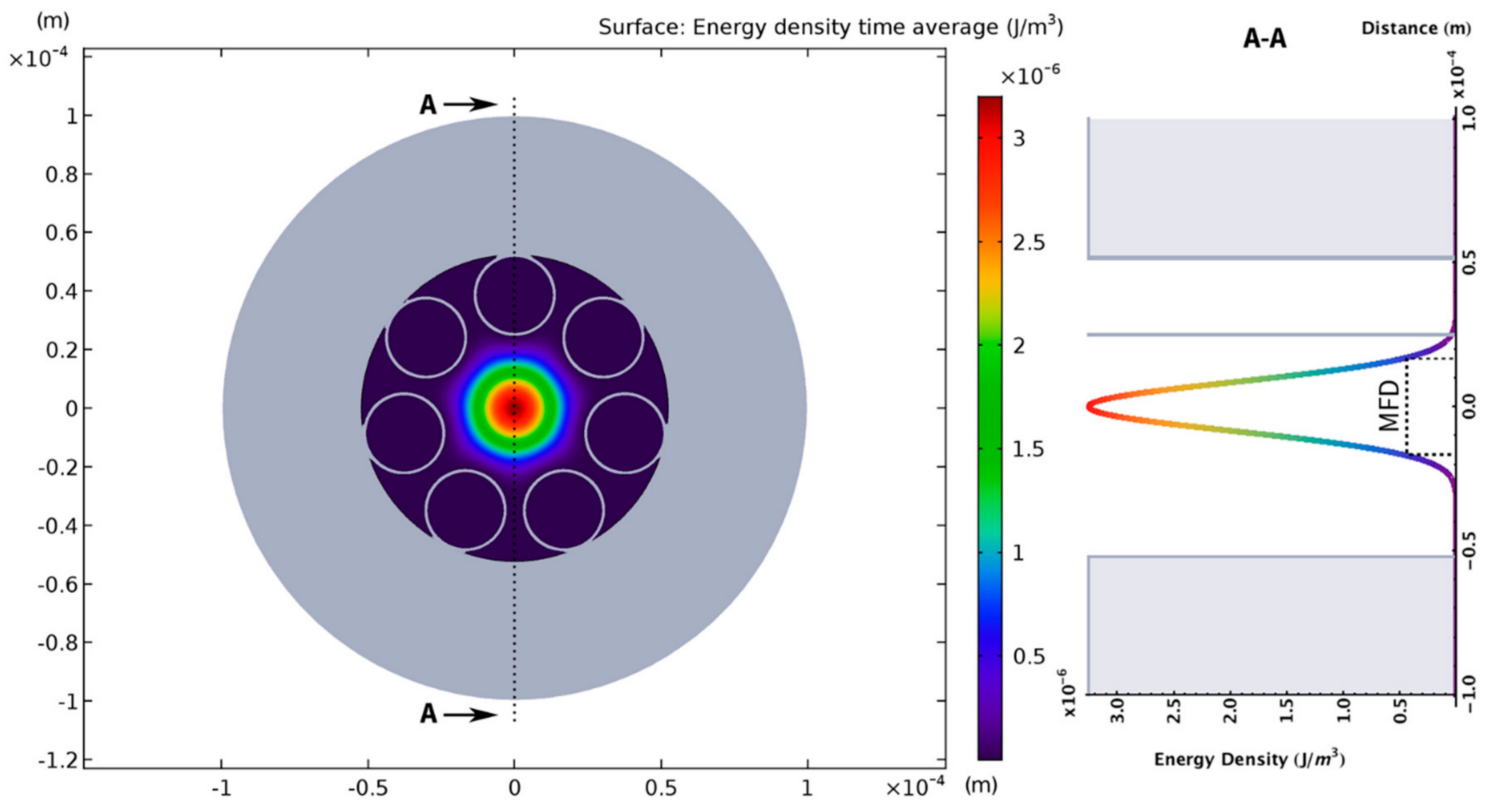

Figure 2. Schematic light field intensity overlapping with the glass structure in an antiresonant fiber design.

The coupling efficiency can be calculated theoretically by calculating the overlap of coupled free-space mode and the fiber eigenmodes. Variations can also be introduced and the results can be compared to experimental results using low-power beams (see Figure 3). 


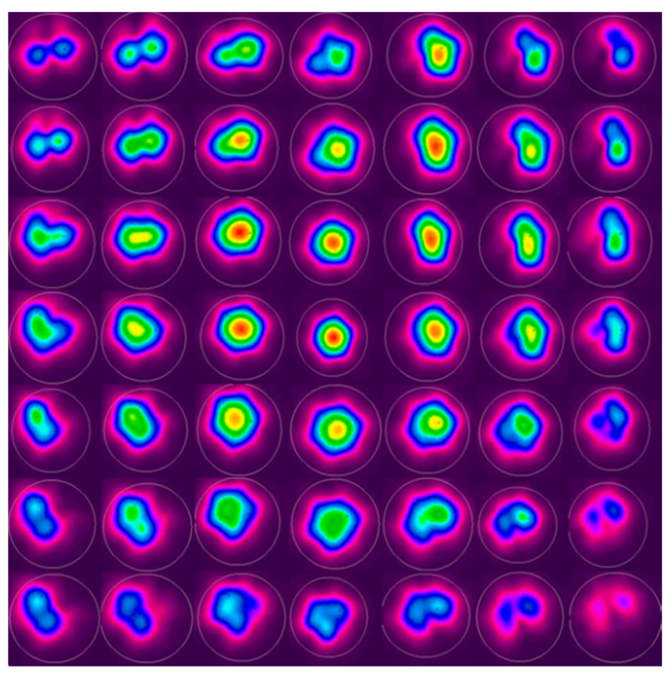

Figure 3. Transmitted mode content under varying coupling conditions of a hollow core fiber.

Typically, high-power pulses travelling along the fiber will often have enough energy to interact with the gas inside the hollow core, which will alter the pulse characteristiocs [13-15]. In order to reduce these effects, the fiber material can be filled with other gases or kept at a vacuum to significantly reduce any gas interaction. However, the fiber itself will still have a small waveguide dispersion, which will lead to pulse broadening and chirping. Therefore, if very short pulses below $100 \mathrm{fs}$ are demanded, the dispersive interaction inside the fiber has to be taken into account and can be precompensated in the compressor of the coupled laser.

Fibers for infrared (IR) light delivery have been produced for a few years, but transmission at shorter wavelengths in the visible or even in the ultraviolet (UV) regime is of high interest. Theoretically, the fundamental guiding mechanism of hollow core fibers allows for structures which transmit in these wavelength range; however, the real-life loss due to scattering is also growing with smaller wavelengths. Due to this, the production tolerances are a key factor when designing fibers in the visible and UV spectral region and currently do not allow for fiber material which can match the attenuation and stability of IR at smaller wavelengths. In general, achieving low-loss and nearly undisturbed beam propagation requires a fiber structure which is optimized for the desired transmission window as well as the production routine.

\subsection{Fiber Integration}

Bringing the hollow core fiber technology to the industry has been a challenging process. Typically, laboratory experiments hold a fiber freely in a tabletop setup with microadjusters for low power, or in a gas cell for tailored nonlinear processes. In both cases, the fiber is prepared for the investigation which is planned. This, of course, differs vastly from using a fiber as a tool, where it has to perform, mostly without preparation, in a more demanding environment.

A special connector, which houses the fiber tip in a sealed environment, has been designed (see Figure 4). The fiber is protected from environmental influences but can also be filled with gas or evacuated. Differential pumping schemes are also possible by applying different pressures at each side of the laser light cable. Standard safety features, such as a fiber continuity monitoring, are also included, as well as the possibility for active cooling of the connector when used with very high power. The conduit is designed with continuous use in demanding application in mind and can protect the fiber even after years of continuous bending. The fiber itself is prealigned to very few micron precision and can be exchanged in few minutes by the use of a flange connector which is easy to adapt. 


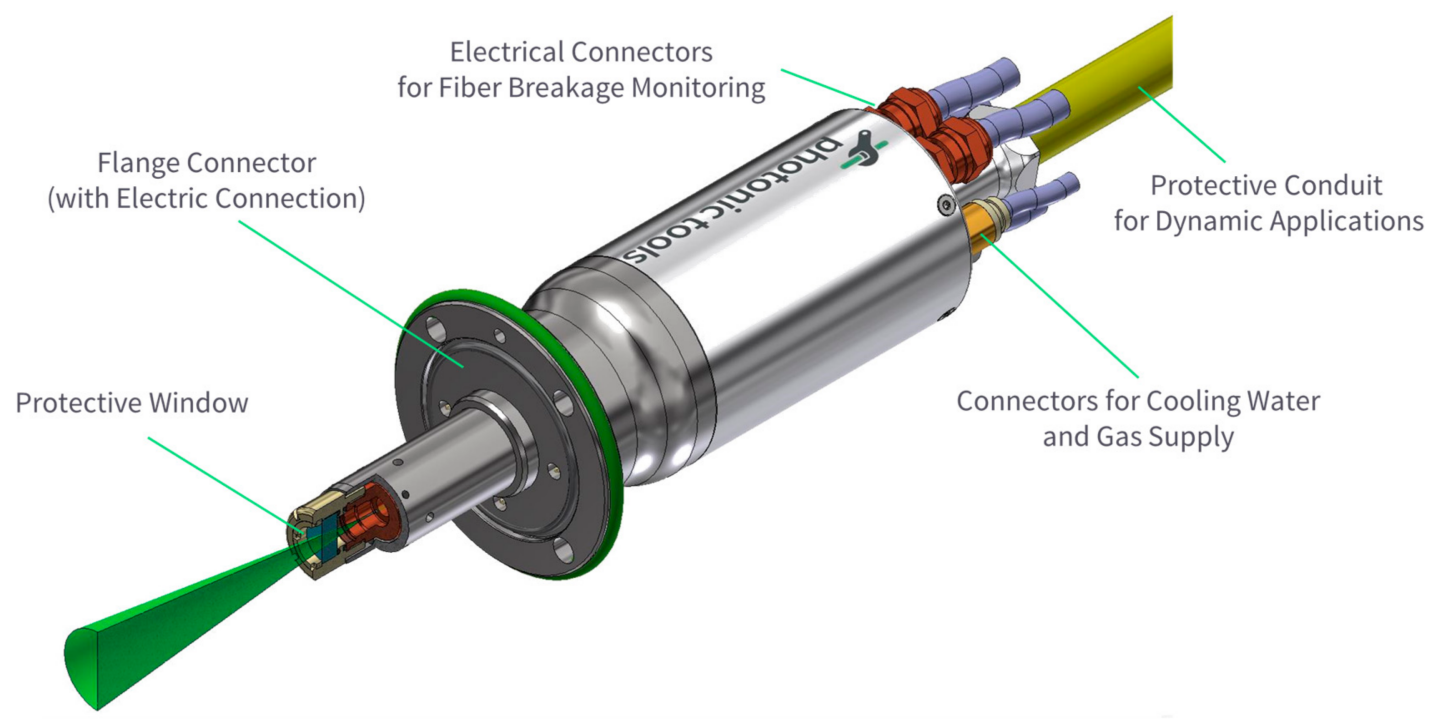

Figure 4. Ultrafast laser light cable connector with connections for water cooling, evacuation and safety circuits.

Together with the laser light cable, a modular system, composed of components specially designed for ultrafast laser beam delivery and applications, has also been introduced (see Figure 5) [16]. These components include a coupling unit, which can accept a wide range of laser beams and adapt them to be optimally coupled into the fiber. The unit uses a flexible modular design and can also be integrated into a laser head. On the output side, different beam shaping tools are available, ranging from collimation units, which prepare a requested beam size, to complex processing heads with diagnostics, observation and variable beam shaping.

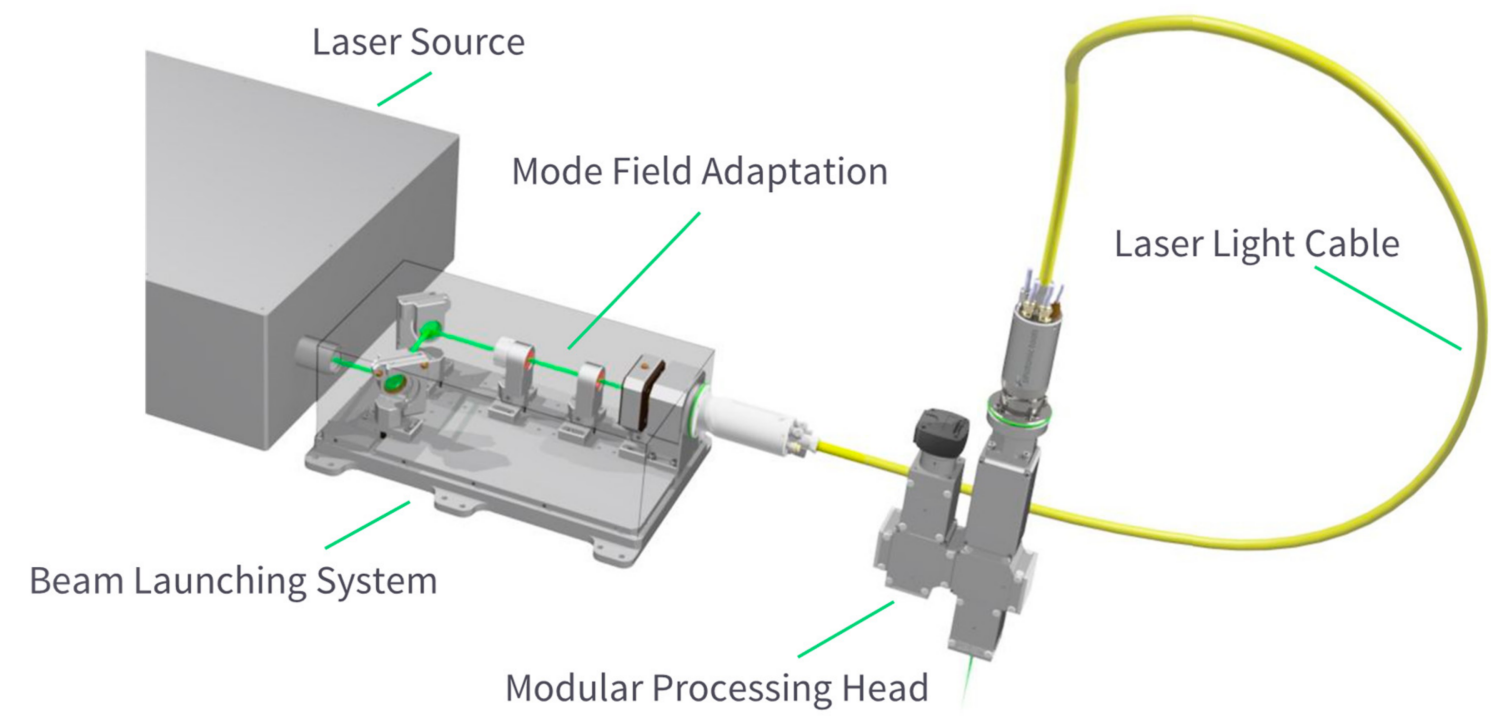

Figure 5. Ultrafast beam delivery system with a beam launching system (BLS) composed of a mode field adaptation, a laser light cable (LLK) and a modular processing head (MPH).

Whilst the ultrafast laser beam can also be delivered to the workpiece by free-space beam delivery components, transporting the laser beam by fiber offers several advantages for the laser user.

Integrating the laser source into the application system or machine will be much simpler, because the flexible beam delivery enables the laser source to be installed separately from the optics, which focuses the beam into the workpiece. For example, this allows for a much simpler mechanical 
structure and lower moved masses by simply routing the laser light cable, instead of a fixed beam path using multiple mirrors.

Additionally, the service of the system is becoming much simpler because of the reduced number of components in the beam path, and the possibility of installing and exchanging a laser light cable with no or only very little beam alignment. The latter is possible since the fiber is positioned very accurately in the laser light cable connector and therefore provides an excellent optomechanical reference for the laser beam.

In summary, this leads to a lower cost of ownership for the complete laser system not only in capital investment but also running cost. The additional positive side effect is an improved production uptime of the complete laser application system, which is mainly driven by less complexity and simplified serviceability.

The combination of optical design tailored specifically for the use in conjunction with hollow core fibers of different designs as well as rigorous characterization has led to a design, which shows remarkable stability under varying conditions and with most laser sources.

\section{Results}

\subsection{Performance}

Numerous integrations of the ultrafast beam delivery system over the years, with varying laser pulse characteristics, have been performed. Peak powers of up to a gigawatt and an average power of $200 \mathrm{~W}$ have been transmitted with an efficiency between $85 \%$ and $93 \%$, depending on the length of the fiber and the beam quality of the laser coupled into it (see Figure 6 for a selection).
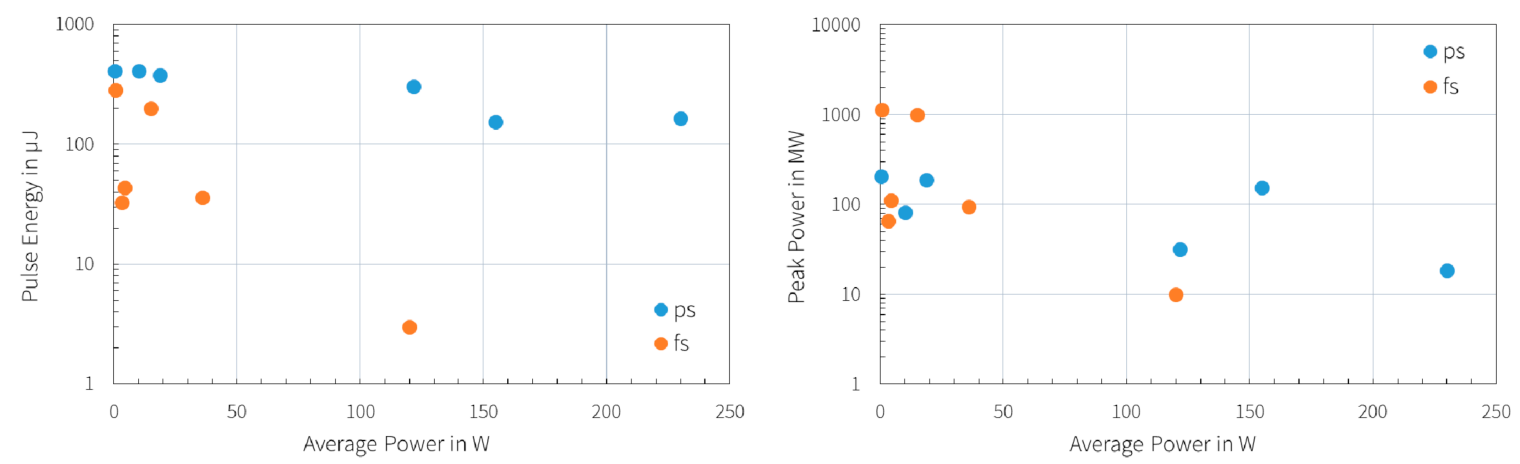

Figure 6. Overview of pulsed laser values which could be coupled and transmitted successfully using the flexible beam delivery. Each dot represents a parameter pair of the laser source. Picosecond and femtosecond laser systems are separately marked.

Higher-modes present in the coupled laser beam are more likely to excite higher-order modes inside the fiber, which reduces transmission and stability. Different fiber designs can influence this behavior in different strengths. An example for a stable result can be seen in Figure 7, where the residual movement of the center of intensity of the laser beam at the workpiece is shown. It was measured while performing strong bending and twist movement of the laser light cable. As can be seen, the residual movement of the beam, even under strong bending of the laser light cable, only amounts to very few percentages of the focus spot size, which hardly has any impact on the typical laser application. 


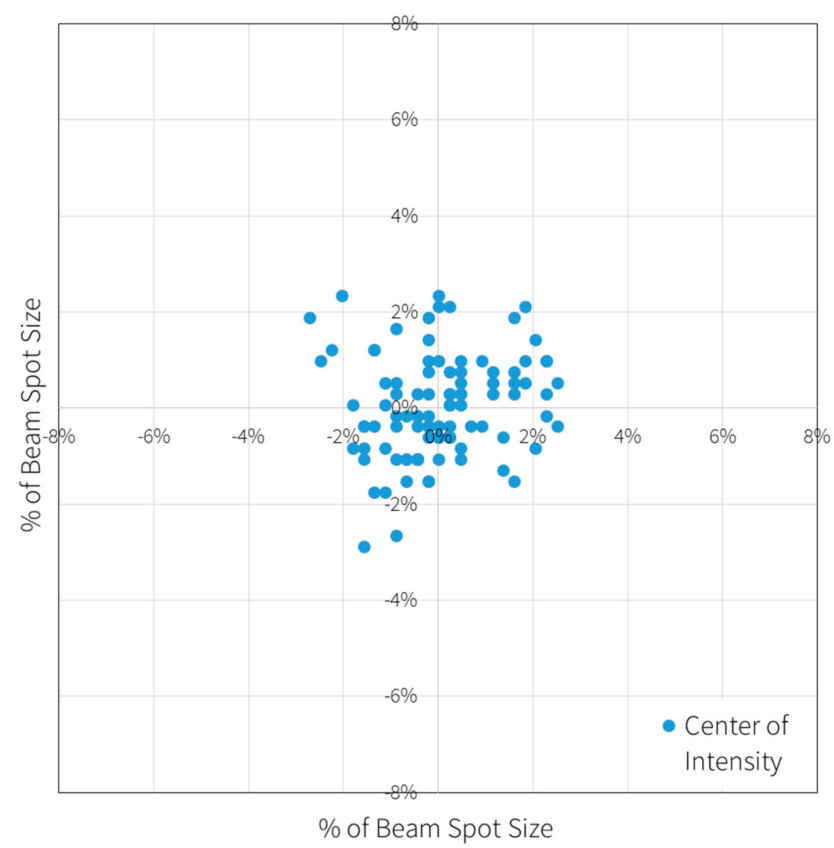

Figure 7. Movement of the focus spot relative to the beam size at the fiber output while moving the fiber in an application.

\subsection{Application}

Hollow core fibers can transmit ultrafast beams nearly undisturbed. Due to the wide range of pulse paramters possible, different residual influences may affect the transmitted beam slightly. To determine their influence in typical machining applications, many comparative application studies have been conducted. These include sensing, cutting, drilling, ablation and surface structuring and modification.

The key parameters for these applications are the focus intensity and focus shape as well as the pulse shape. All of these can be controlled very precisely when using a hollow core fiber for beam delivery. The output of the fiber is providing a near single-mode beam with minimal fluctuations and fixed positions, which allows for a precise processing.

For this investigation, the fiber beam delivery was integrated in different applications and the results were compared qualitatively with free-space beam paths.

Firstly, cutting results from nitinol stent cutting are shown (see Figure 8). The process parameters are shown in Table 1.

Table 1. Processing parameters of nitinol cutting.

\begin{tabular}{|c|c|c|}
\hline Parameters & Value & Unit \\
\hline Laser source & \multicolumn{2}{|c|}{ Amplitude Satsuma } \\
\hline Pulse length & 350 & fs \\
\hline Power & 5 & W \\
\hline Repetition rate & $0-2$ & $\mathrm{MHz}$ \\
\hline Beam delivery & \multicolumn{2}{|c|}{ Photonic Tools } \\
\hline LLK length & 3 & $\mathrm{~m}$ \\
\hline Collimation & 100 & $\mathrm{~mm}$ \\
\hline Air pressue in fiber & $5-1000$ & mbar \\
\hline Workpiece & \multicolumn{2}{|c|}{ Nitinol } \\
\hline Wavelength & 1030 & $\mathrm{~nm}$ \\
\hline Polarization & circular & \\
\hline Repetition rate & 300 & $\mathrm{kHz}$ \\
\hline Pulse energy & $\sim 8$ & $\mu \mathrm{J}$ \\
\hline Spot size & $\sim 12$ & $\mu \mathrm{m}$ \\
\hline
\end{tabular}




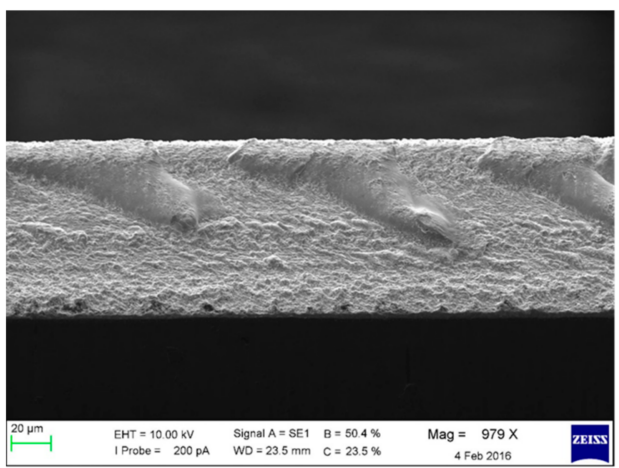

freespace beam

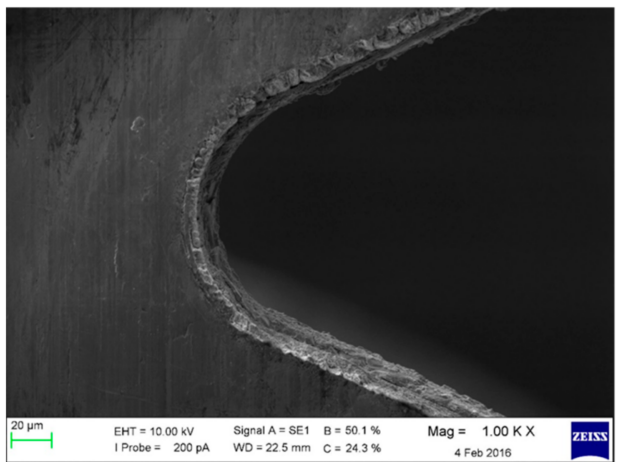

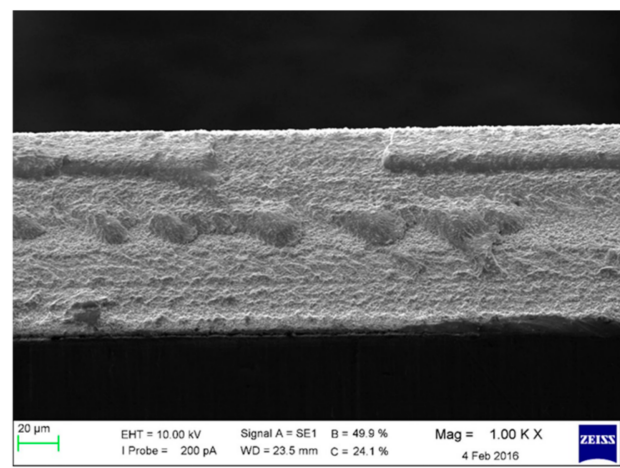

flexible beam delivery

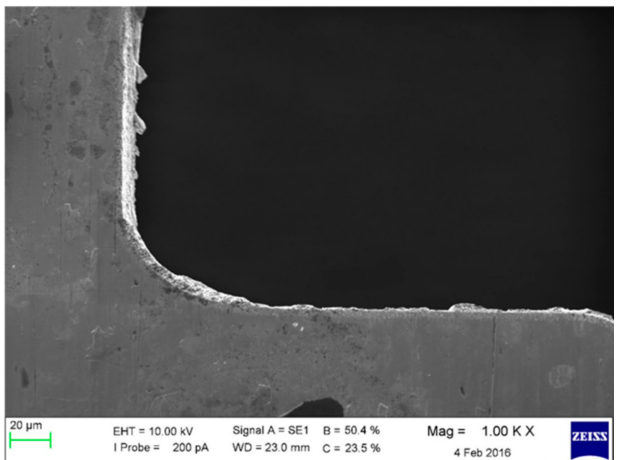

Figure 8. Comparison of cutting results. Free-space beam delivery on the left and fiber beam delivery on the right (in co-operation with femtos $\mathrm{GmbH}$ ).

The comparison shows very similar results with the two different beam delivery methods. The quality of the cutting edge is of the same quality and the final products could be further processed without any alteration.

Next, results from silicon surface modification are presented (see Figure 9). A square was scanned with varying process parameters while incorporating a free-space or fiber beam delivery in the beam path to the galvanometer scanner. See Table 2 for the corresponding process parameters. Additionally, the fiber could be used to widen the spectrum of the pulse due to self-phase modulation, controlled by adjusting the pressure inside the fiber. The spectral width of the pulse was measured with a spectrometer.

Table 2. Processing parameters of silicon surface modification.

\begin{tabular}{|c|c|c|}
\hline Parameters & Value & Unit \\
\hline Laser source & \multicolumn{2}{|c|}{ Amplitude S-Pulse } \\
\hline Pulse length & $750^{1}$ & fs \\
\hline Power & 4 & W \\
\hline Repetition rate & $0-300$ & $\mathrm{kHz}$ \\
\hline Beam delivery & \multicolumn{2}{|c|}{ Photonic Tools } \\
\hline LLK length & 2.5 & $\mathrm{~m}$ \\
\hline Collimation & 100 & $\mathrm{~mm}$ \\
\hline Air pressue in fiber & $5-1000$ & mbar \\
\hline Workpiece & \multicolumn{2}{|c|}{ Polycrystalline silicon } \\
\hline Wavelength & $\begin{array}{c}1028 \pm 3 \\
1030 \pm 30\end{array}$ & $\mathrm{~nm}$ \\
\hline Polarization & linear & \\
\hline Repetition rate & 100 & $\mathrm{kHz}$ \\
\hline Pulse energy & 12.5 & $\mu \mathrm{J}$ \\
\hline Spot size & 16 & $\mu \mathrm{m}$ \\
\hline Scan speed & 4 & $\mathrm{~mm} / \mathrm{s}$ \\
\hline
\end{tabular}




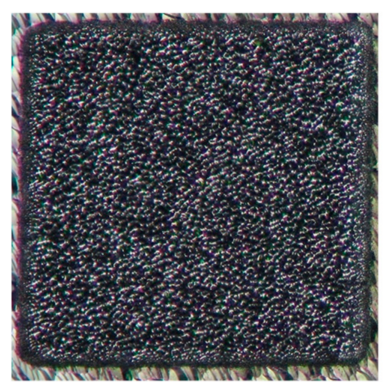

freespace beam

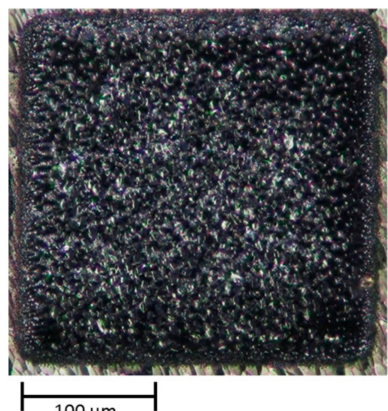

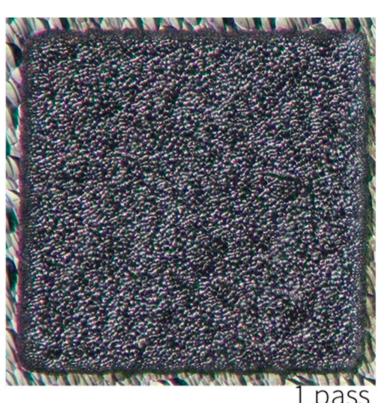

flexible beam delivery

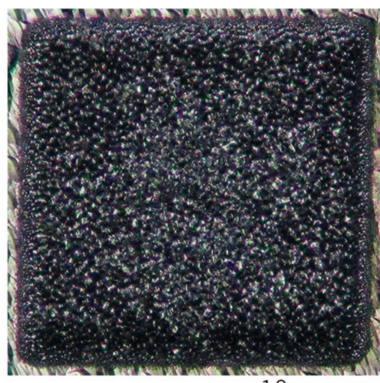

10 passes

Figure 9. Comparison of surface modification on polychristalline silicon. A single pass of the laser over the area is shown at the top and 10 consecutive passes are shown at the bottom. Free-space beam delivery on the left and fiber beam delivery on the right (in cooperation with Laserzentrum Hannover).

Again, the comparison revealed almost no visible difference between the two beam delivery solutions. The structures, which depended critically on the pulse parameters in size and depth, could be reproduced perfectly with 1 pass over the surface as well as 10 consecutive scans over the same area.

Additionally, a unique feature of the fiber beam delivery was tested and deliberate self-phase modulation could be introduced to the transmitted pulses in varying strengths. Strong increases in ablation depth and structure size were observed when using spectrally broadened pulses (see Figure 10). The pulse bandwidth change from $6 \mathrm{~nm}$ to $60 \mathrm{~nm}$ revealed a nearly doubled ablation rate in the application.
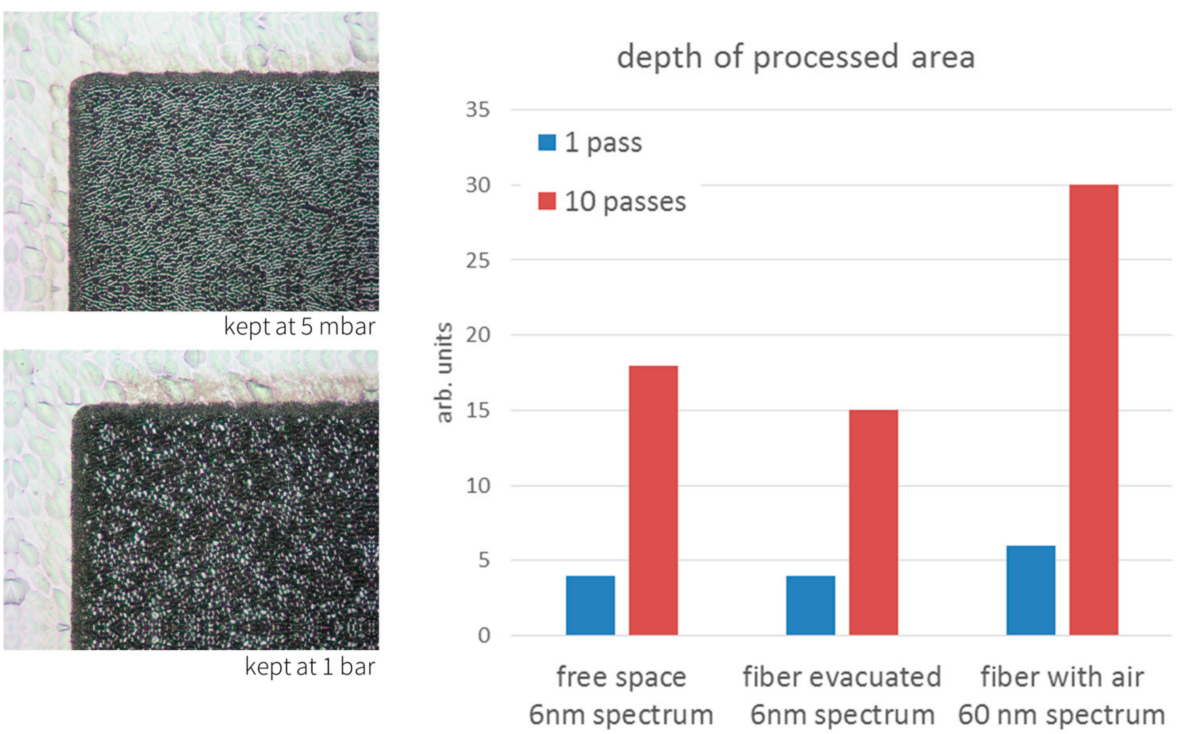

Figure 10. Surface modification on polychristalline silicon with spectrally broadened pulses. Ablation depths and structure sizes with pulse bandwidth change from $6 \mathrm{~nm}$ to $60 \mathrm{~nm}$ were compared. 


\section{Conclusions}

The use of hollow core fiber for transmission of ultrafast laser pulses in an industrial environment has been shown for a few years in the IR wavelength of YAG laser systems. The completely fiber-based beam delivery system introduced by Photonic Tools already addresses most of the application scenarios, which are actively used. Robot arm applications with hollow core fibers have also been shown. The comparison of free-space beam delivery and fiber-based solutions shows little to no difference in the application results. On the contrary, it can even offer opportunities to increase throughput by using the nonlinear effects inside the hollow core fiber. In a suitable integration, as offered by Photonic Tools, they can be adjusted precisely.

The optimal fiber structure for industrial appplication is still being improved and optimized, so understanding the requirements from applications and characterizing hollow core fibers very rigorously are an essential part of integrating hollow core material into a demanding environment. Recent fiber developments in the green and UV spectral regions show promise for future applications, but cannot reach the same level of performance in high-power applications at the moment.

Taking hollow core fiber out of the scientific environment and tweaking it to perform under diverse conditions is a continuous process, which has made tremendous progress in recent years. Many industrial ultrafast applications can already use the advantages a fiber beam delivery can offer in day-to-day usage with high reliability and unaltered results.

Author Contributions: Conceptualization, S.E. and B.W.; Data curation, S.E.; Funding acquisition, B.W.; Investigation, S.E.; Project administration, B.W.; Resources, B.W.; Supervision, B.W.; Writing-original draft, S.E.; Writing-review \& editing, S.E. and B.W.

Funding: This research was funded by the Bundesministerium für Bildung und Forschung, grant numbers [13N13920, 13N13924, 02P14K500].

Acknowledgments: We thank J. Düsing from Laserzentrum Hannover and B. Schöps from femtos GmbH for their cooperation and advise while performing and interpreting the application results.

Conflicts of Interest: The authors declare no conflict of interest. The funders had no role in the design of the study; in the collection, analyses, or interpretation of data; in the writing of the manuscript, and in the decision to publish the results.

\section{References}

1. Sugioka, K.; Cheng, Y. Ultrafast lasers-Reliable tools for advanced materials processing. Light Sci. Appl. 2014, 3, e149. [CrossRef]

2. Cregan, R.F.; Mangan, B.J.; Knight, J.C.; Birks, T.A.; Russell, P.S.J.; Roberts, P.J.; Allan, D.C. Single-mode photonic band gap guidance of light in air. Science 1999, 285, 1537-1539. [CrossRef] [PubMed]

3. Debord, B.; Alharbi, M.; Bradley, T.; Fourcade-Dutin, C.; Wang, Y.Y.; Vincetti, L.; Gérôme, F.; Benabid, F. Hypocycloid-shaped hollow-core photonic crystal fiber part I: Arc curvature effect on confinement loss. Opt. Express 2013, 21, 28597-28608. [CrossRef] [PubMed]

4. Litchinitser, N.M.; Abeeluck, A.K.; Headley, C.; Eggleton, B.J. Antiresonant reflecting photonic crystal optical waveguides. Opt. Lett. 2002, 27, 1592-1594. [CrossRef] [PubMed]

5. Yu, F.; Knight, J.C. Spectral attenuation limits of silica hollow core negative curvature fiber. Opt. Express 2013, 22, 21466-21471. [CrossRef] [PubMed]

6. Alharbi, M.; Bradley, T.; Debord, B.; Fourcade-Dutin, C.; Ghosh, D.; Vincetti, L.; Gérôme, F.; Benabid, F. Hypocycloid-shaped hollow-core photonic crystal fiber Part II: Cladding effect on confinement and bend loss. Opt. Express 2013, 21, 28609-28616. [CrossRef] [PubMed]

7. Finger, M.A.; Joly, N.Y.; Weiss, T.; Russell, P.S.J. Accuracy oft he capillary approximation for gas-filled kagomé-style photonic crystal fiber. Opt. Lett. 2014, 39, 821-824. [CrossRef] [PubMed]

8. Carter, R.M.; Yu, F.; Wadsworth, W.J.; Shephard, J.D.; Birks, T.; Knight, J.C.; Hand, D.P. Measurement of resonant bend loss in anti-resonant hollow core optical fiber. Opt. Express 2017, 25, 20612-20621. [CrossRef] [PubMed]

9. Michieletto, M.; Lyngsø, J.K.; Jakobsen, C.; Lægsgaard, J.; Bang, O.; Alkeskjold, T.T. Hollow-core fibers for high power pulse delivery. Opt. Express 2016, 24, 7103-7119. [CrossRef] [PubMed] 
10. Ermolov, A.; Mak, K.F.; Frosz, M.H.; Travers, J.C.; Russell, P.S.J. Supercontinuum generation in the vacuum ultraviolet through dispersive-wave and soliton-plasma interatction in a noble-gas-filled hollow-core photonic crystal fiber. Phys. Rev. A 2015, 92, 033821. [CrossRef]

11. Benabid, F.; Knight, J.C.; Antonopoulos, G.; Russell, P.S.J. Stimulated raman scattering in hydrogen-filled hollow-core photonic crystal fiber. Science 2002, 298, 399-402. [CrossRef] [PubMed]

12. Smith, A.V.; Do, B.T.; Hadley, G.R.; Farrow, R.L. Optical damage limits to pulse energy from fibers. IEEE J. Sel. Top. Quantum Electron. 2009, 15, 153-158. [CrossRef]

13. Bhagwat, A.R.; Gaeta, A.L. Nonlinear optics in hollow-core photonic bandgap fibers. Opt. Express 2008, 16, 5035-5047. [CrossRef] [PubMed]

14. Chang, W.; Nazarkin, A.; Travers, J.C.; Nold, J.; Hölzer, P.; Joly, N.Y.; Russell, P.S.J. Influence of ionizsation on ultrafast gas-based nonlinear fiber optics. Opt. Express 2011, 19, 21018-21027. [CrossRef] [PubMed]

15. Mousavi, S.A.; Mulvad, H.C.H.; Wheeler, N.V.; Horak, P.; Hayes, J.; Chen, Y.; Bradley, T.D.; Alam, S.; Sandoghchi, S.R.; Richardson, D.J.; et al. Nonlinear dynamic of picosecond pulse propagation in atmospheric air-filled hollow core fibers. Opt. Express 2018, 26, 8866-8882. [CrossRef] [PubMed]

16. Funck, M.C.; Eilzer, S.; Wedel, B. Ultrafast beam delivery: System technology and industrial application. In Proceedings of the High-Power Laser Materials Processing: Applications, Diagnostics, and Systems VI, San Francisco, CA, USA, 28 January-2 February 2017; p. 100970L.

(C) 2018 by the authors. Licensee MDPI, Basel, Switzerland. This article is an open access article distributed under the terms and conditions of the Creative Commons Attribution (CC BY) license (http:/ / creativecommons.org/licenses/by/4.0/). 